\title{
MICROMACHINING OF ELECTRET MATERIALS, ADVANTAGES AND POSSIBILITIES
}

\author{
J.A. Voorthuyzen and P. Bergveld
}

Twente University, P.O. Box 217, 7500 AE Enschede

The Netherlands

\begin{abstract}
In this paper we describe a process for the micromachining of organic electret materials like Teflon-FEP. We have used photolithography and plasma etching, by which electrets can be etched selectively.

The advantages of, in such a way micromachined electrets are numerous. Miniature sensors like microphones and pressure sensors, containig local areas with Teflon, have been realized in our laboratories. The process can also be used for electret research. An example is the overall reduction of electret film thickness, by which we have determined the average penetration depth of accelerated electrons in Teflon-FEP.
\end{abstract}

\section{INTRODUCTION}

The application of carbon-fluor polymers, for electrets is well known. Teflon-FEP has a resistivity of $10^{21} \Omega \mathrm{cm}$ and a reported charge-decay time of more than 10 years [1]. The use of Teflon-PTFE for electret and piezoelectric applications is also well known.

The electret properties of such polymers appear to depend on the film thickness. Useful values, and commercially available are 12.5 and $25 \mu \mathrm{m}$ [1]. In many applications the thickness of the foil and the size of the structures to be realized are not very critical.

However, in the Biosensor Research Group of Twente University we are considering the application of Teflon foils in the development of microtransducers with submillimeter dimensions [2]-[6]. In that case the lateral dimensions of the foil as well as its thickness are very important and should be controlled in a well-defined way.

A very useful tool in the realization of microsensors is photolithography. This technique enables us to pattern thin films in many different profiles. The process consists of several steps, as schematically drawn in figure 1 and described below. 


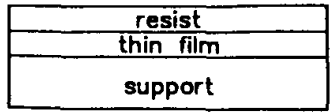

o) resist spinning

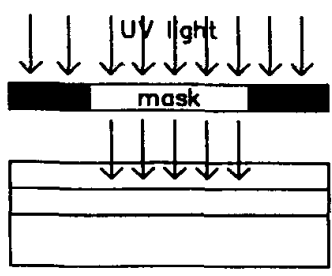

b) UV exposure

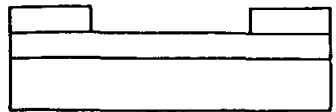

c) resist development

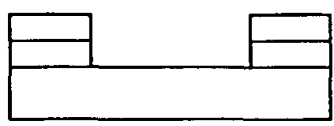

d) thin film etching

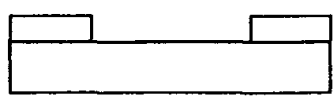

e) cleaning

Figure 1: consecutive steps in photolithography.

\section{PHOTOLITHOGRAPHY}

The film, deposited on a support (usually a silicon wafer ) is spin-coated with a 2-3 $\mu \mathrm{m}$ thick layer of photoresist. The resist is pre-polymerized during 20 minutes at $90{ }^{\circ} \mathrm{C}$. The wafer is positioned under a mask and then exposed to UV-light, causing exposed parts of the resist to depolymerize (for so-called positive resist) and non-exposed parts to remain unchanged. Then the wafer is developed and the UV-radiated resist is solved. The wafer is post-baked to complete polymerization and then placed in an etching liquid or gas, specific for the material of the thin film. Finally the resist layer itself is removed and the locally etched wafer is cleaned.

\section{ETCHING OF CARBON-FLUOR POLYMERS}

The use of wet chemical etchants for photolithography is rather cheap and easy. For many materials selective etchants are known.

However, for carbon-fluor polymers such etchants are not known. In literature the use of a sodium dispersion suspended in naphtalene has been reported for the etching of Teflon, but it appears only to remove fluorine atoms from the surface, facilitating its bonding to other materials, and not to etch with a reasonable speed [7].
A quite
differen
possioility
is to use
a plasma. 
Generally a plasma is created by applying a high voltage between two electrodes in a vacuum chamber in which a certain gas is injected. For several materials, like silicon and silicon dioxide, it has been found that plasma's from $\mathrm{CCl}_{4}$ and $\mathrm{CH}_{3} \mathrm{~F}$ respectively can be used for the selectively plasma etching of these materials. It has also been found that an $\mathrm{O}_{2}$ plasma can be used for the plasma etching of Teflon [8].

In a plasma different etching mechanisms may be active, depending on the processing parameters like power-density, gas flow and substrate temperature. The etching characteristics, like selectivity (the preferential etching of one material) and anisotropy (the etching speed lateral to the substrate differs from that in perpendicular direction) for those mechanisms are quite different:

1) Physical etching:

In the plasma, ions are created and accelerated in the applied electric field. They strike the surface of the thin film which will be damaged and is thus etched. Due to the acceleration of ions in one direction it is anisotropic, causing rather perpendicular etched profiles if a patterned masking layer is used. However, this mechanism is not very selective, causing that layers of different materials are etched with comparable speed.

2) Chemical etching:

In the plasma also radicals of the gas are formed which may chemically react and have a preferential etching behaviour for one specific material. The transport of radicals in the plasma is not determined by the applied electric field, and thus will result in isotropic etching causing considerable underetching.

3) Physically enhanced chemical etching:

A combination of both processes may of course also occur. The chemical etching with radicals may be enhanced by ion bombardment. It will result in more underetching than physical etching, but is also more selective. The exact properties depend on the relative influence of both mechanisms on the etching characteristics.

\section{EXPERIMENTAL}

We have investigated the etching characteristics of Teflon FEP in an oxygen plasma in a barrel reactor and a plasma-etcher.

In a barrel reactor the applied electric field is parallel to the thin film surface and in a plasma etcher perpendicular to it. This means that in a barrel reactor the etching will be of chemical origin, while in a plasma etcher 
all mechanisms mentioned above may occur.

The etching of Teflon in the barrel reactor appeared to be negligibe, although in the same reactor layers of resist have been etched favourably with considerable speed (about 0.1-0.2 $\mu \mathrm{m} / \mathrm{min}$.). In the plasma-etcher Teflon has been etched with a speed of about $0.15-0.30 \mu \mathrm{m} / \mathrm{min}$.), which implies an overall etching time of about $80-40 \mathrm{~min}$ for $12.5 \mu \mathrm{m}$ foils. The underetching appeared to be less than the intial thickness of the Teflon layer. The etching speed can be controlled by the power-density. In general it may be attractive to use the highest etching speed as possible. However, we have found that in that case a residual layer of unknown composition is formed, that can not be removed. Using an etching speed of $0.15 \mu \mathrm{m} / \mathrm{min}$. this effect was not observed.

To pattern a Teflon layer a mask is required. In this case photoresist could not be used, because it is also etched in an $\mathrm{O}_{2}$ plasma with at least the same speed as Teflon.We have found that a $0.2 \mu \mathrm{m}$ thick layer of aluminium, deposited by evaporation and patterned by photolithography can be used succesfully. It will be clear that after the Teflon etching process, the aluminium mask has to be removed.

Considering the rather high selectivity of the etch-process between Teflon and aluminium, the observed under-etching and the low etching speed in a barrel reactor, we conclude that the etching mechanism of Teflon in an oxygen plasma is physically enhanced chemical etching.

\section{OTHER APPLICATIONS}

We have described a process that enables to shape Teflon layers for use in microsensors e.g. for blood pressure and acoustic pressure, as described elsewhere [2]-[6].

The same process can also be used for determining the average penetration depth of electron beams in flat Teflon samples. We have realized Teflon samples on a conductive support with different thicknesses by changing the etch-time. The thickness has been measured by determining the weight loss after etching. In all samples the same amount of electric charge has been injected by a SEM. This implies that the surface potential linearly depends on the electret thickness. Plotting the surface potential (as measured by a Monroe Probe) of the various electrets versus the electret thickness, the intersection with the $\mathrm{x}$-axis yields the average penetration depth, as shown in figure 2 . The acceleration voltage was 15 $\mathrm{kV}$. We observe an average penetration depth of $2.5 \mu \mathrm{m}$, which corresponds rather well to the value reported elsewhere [1]. 


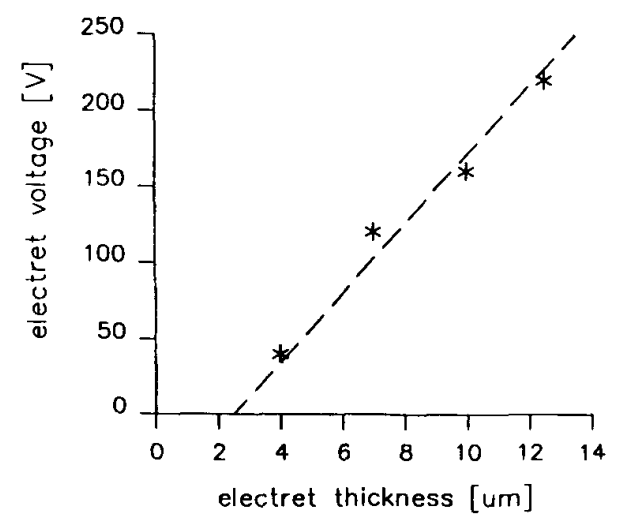

Figure 2: measured electret surface potential as a fucntion of electret thickness.

\section{REFERENCES}

[1] G.M. Sessler, "Topics in applied physics, vol. 33; Electrets", Springer-Verlag, Berlin, 1980.

[2] J.A. Voorthuyzen and P. Bergveld, "Theoretical considerations in the design of integrated semiconductor sensors applying electrets", IEEE Trans. Electron Dev., vol. ED-32, no. 7, pp. 1185-1190, July 1985.

[3] A.J. Sprenkels, J.A. Voorthuyzen and P. Bergveld, "A theoretical analysis of the electret air-gap field effect structure for sensor application", Sensors and Actuators, vol. 9, pp. 59-72, 1986.

[4] J.A. Voorthuyzen, "The PRESSFET, an integrated electret-MOSFET structure for application as a catheter-tip blood pressure sensor", Thesis, Twente University, 1986.

[5] A.J. Sprenkels, "A silicon subminiature electret microphone ", Thesis, Twente University, 1988.

[6] A.J. Sprenkels and P. Bergveld, "The use of silicon technology for an electret microphone construction", This proceedings.

[7] S.J. Updike, M.C. Shults and M. Busby, "Continuous glucose monitor based on an immobilized enzyme electrode detector", J. Lab. Clin. Med., vol. 93, no. 4, pp. 518-527, 1979.

[8] L.A. Perderson, "Structural composition of polymers relative to their plasma etch characteristics", J. Electrochem. Soc., vol. 129, no. 1, pp. 205-208, Jan. 1982. 